\title{
Collagen birefringence assessment in heart chordae tendineae through PS-OCT
}

\author{
Eusebio Real*a ${ }^{* a} J^{\prime}$ osé M. Revuelta ${ }^{\mathrm{b}}$, Nieves González-Vargas ${ }^{\mathrm{a}}$, Alejandro Pontón ${ }^{\mathrm{c}}$, Marta Calvo- \\ Díez ${ }^{\mathrm{c}}$, José M. López-Higuera ${ }^{\mathrm{a}, \mathrm{d}}$, Olga M. Conde ${ }^{\mathrm{a}, \mathrm{d}, \mathrm{e}}$ \\ ${ }^{a}$ Photonics Engineering Group, Dep. TEISA, University of Cantabria, Plaza de la Ciencia s/n, 39005 \\ Santander, Spain; ${ }^{\mathrm{b}}$ Medical and Surgical Sciences Department. Faculty of Medicine. University of \\ Cantabria, Av Herrera Oria, s/n, 39011 Santander, Spain; ${ }^{\mathrm{c}}$ Cardiovascular Surgery Service. Marqués \\ de Valdecilla University Hospital, Av. Valdecilla, 25, 39008 Santander, Spain; ${ }^{\mathrm{d}}$ CIBERBBN, Av. \\ Monforte de Lemos 3-5, 28029 Madrid, Spain; ${ }^{\mathrm{e}}$ IDIVAL, Avenida Cardenal Herrera Oria s/n, \\ 39011 Santander
}

\begin{abstract}
Degenerative mitral regurgitation is a serious and frequent human heart valve disease. Malfunctioning of this valve brings the left-sided heart through a significant increase of pressure and volume overload. Severe degenerative mitral incompetence generally requires surgical repair or valve replacement with a bioprosthesis or mechanical heart valve.

Degenerative disease affects the leaflets or/and the chordae tendineae, which link both leaflets to the papillary muscles. During mitral valve surgical repair, reconstruction of the valve leaflets, annulus and chordae are provided to prevent postoperative recurrence of valve regurgitation. The operative evaluation of the diseased and apparently normal chordae tendineae mainly depends of the surgeon's experience, without any other objective diagnosis tool.

In this work, PS-OCT (Polarization Sensitive-Optical Coherence Tomography) is applied for the first time to evaluate the pathological condition of human chordae coming from the mitral valve. It consists on a prospective study to test the viability of this technique for the evaluation of the collagen core of chords. This core presents a strong birefringence due to the longitudinal and organized arrangement of its collagen bundles. Different densities and organizations of the collagen core translate into different birefringence indicators whose measurement become an objective marker of the core structure.

Ex-vivo mitral degenerative chordae tendineae have been analyzed with PS-OCT. Intensity OCT is used to obtain complementary morphological information of the chords. Birefringence results correlate with the previously reported values for human tendinous tissue.
\end{abstract}

Keywords: PS-OCT, chordae tendineae, tendinous cords, collagen organization, birrefringence, degradation diagnosis

\section{INTRODUCTION}

The chordae tendineae are tendinous chords that connect the mitral and tricuspid valves to the heart papillary muscles [1]. Malfunctioning of the mitral valve system causes valve insufficiency, which increases the blood pressure in the left ventricle in order to provide an efficient cardiac output. The surgical options are the valve replacement and/or the repair of the ruptured or elongated chordae tendineae [2]. Different reconstructive techniques are currently used for chordal reconstruction, predominantly the neochordal replacement with polytetrafluoroethylene suture [2].

Degenerative Disease of the Mitral Valve (DDMV) mostly affects tendinous chords [3,4]. The chords are composed of organized and wavy collagen bundles in the core (dense collagen tissue) and elastin outside the core (spongiosa layer), covered by thin epithelium in the outer part (epithelial flat cells layer) [2,3]. When the chordae are degenerative affected, the dense collagen tissue core presents a loose order and, in some cases, the collagen bundles can completely lose their longitudinal organization [3]. As previously reported [5], different reconstructive techniques are used for mitral valve chordae repair. During intervention, surgeons must evaluate the disease chordae, and also identify the normal functioning chordae tendineae, based mainly to their experience and preoperative echocardiography, without any other objective tool.

Optical Coherence Tomography and Coherence Domain Optical Methods in Biomedicine XXI,

edited by James G. Fujimoto, Joseph A. Izatt, Valery V. Tuchin, Proc. of SPIE Vol. 10053,

$1005334 \cdot$ ㅇ 2017 SPIE · CCC code: 1605-7422/17/\$18 · doi: 10.1117/12.2254303 
Optical Coherence Tomography (OCT) has the ability to obtain accurate images of human cardiac structures. It provides morphological information based on tissue scattering and changes of refractive index. Polarization Sensitive OCT (PSOCT) can provide birefringence indicators through phase retardation measurement in the tissue under analysis. The chordae tendineae are formed mainly of collagen bundles organized longitudinally, being a strongly birefringent material. This birefringence is a function of the organization of the collagen in the core of the chord, presenting measurable differences when this structure is modified due to degeneration [6].

\section{MATERIALS AND METHODS}

\subsection{Chordae tendineae}

The chordae tendineae analyzed in this work correspond to human ex-vivo specimens of mitral valve chordae. Specimen samples set consist of 9 chords from 5 patients, obtained in the surgery room during mitral valve repair interventions (4 normal, 1 degenerative).

\subsection{Optical Coherence Tomography}

The OCT system used is the swept source OCS1300SS from Thorlabs. The center wavelength is $1325 \mathrm{~nm}$. Penetration is up to $3 \mathrm{~mm}$ in air, with a resolution of $12 \mu \mathrm{m} \times 25 \mu \mathrm{m}$ axial and lateral respectively. The system has a PS-OCT module (PSOCT-1300), that can provide the phase retardation between the vertical and horizontal polarization channels. This phase translation can be later translated into birefringence.

\subsection{Analysis}

Samples are obtained in Marques de Valdecilla University Hospital (Santander, Spain) and measured at the Photonics Engineering Group to be analyzed within 12 hours after surgery. Intensity OCT and polarization sensitive OCT interrogation is performed under conditions of continuous hydration of the samples.

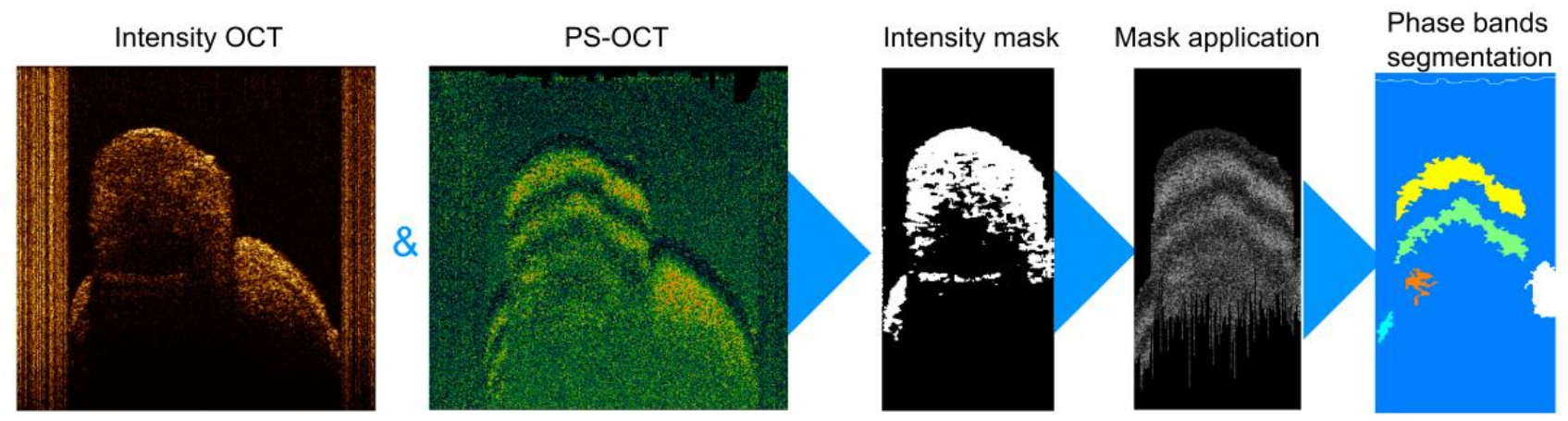

Figure 1. Procedure to obtain the phase retardation bands for birefringence estimation.

a) Intensity and phase retardation OCT images: Intensity OCT represents backscattering intensity due to morphology. PS-OCT represents the phase retardation from $0^{\circ}$ to $180^{\circ}$, being noisier due to random phase fluctuations.

b) Chordae segmentation: Intensity OCT images are used to obtain a precise segmentation of the region under analysis on each chord. A median filter and Otsu's thresholding procedure [7] are used to produce a segmentation mask.

c) Phase bands segmentation: The mask obtained is applied to the phase retardation image. Again, a median filter, Otsu's method combined with dilation and erode procedures are used to delineate those bands presenting different phase behavior.

d) Birefringence pattern: The dimensions of the bands present in the phase retardation images are due to the birefringence $\delta$ according to following equation [8]:

$$
\delta=\frac{\pi}{k_{0} z}=\frac{\pi \lambda_{0}}{2 \pi z}=\frac{\lambda_{0}}{2 z}
$$

where $z$ is the period of the phase retardation bands and $k_{0}$ the speed of light of a wavelength of $\lambda_{0}$. The refractive index of the chord has to be known to calibrate the OCT axial dimension [9]. In this work, the refractive index has been measured, resulting a mean value $n=1.3897$. 


\section{RESULTS AND DISCUSSION}

When healthy chordae and degenerative chordae are compared, the intensity and retardation phase images of the former (right column of Figure 2) reflect a periodic phase retardation pattern whilst the degenerative specimen (left column of Figure 2) do not exhibit any pattern at all. In the first case, the pattern becomes from the birefringence of the wellorganized structure of collagen that constitutes the chord. However, the physiology of the degenerative chordae shows alterations in the synthesis and deposition of collagen and elastin, disorganization of collagen bundles and rupture of collagen fibers within the core [3] that motivates the birefringence lost.
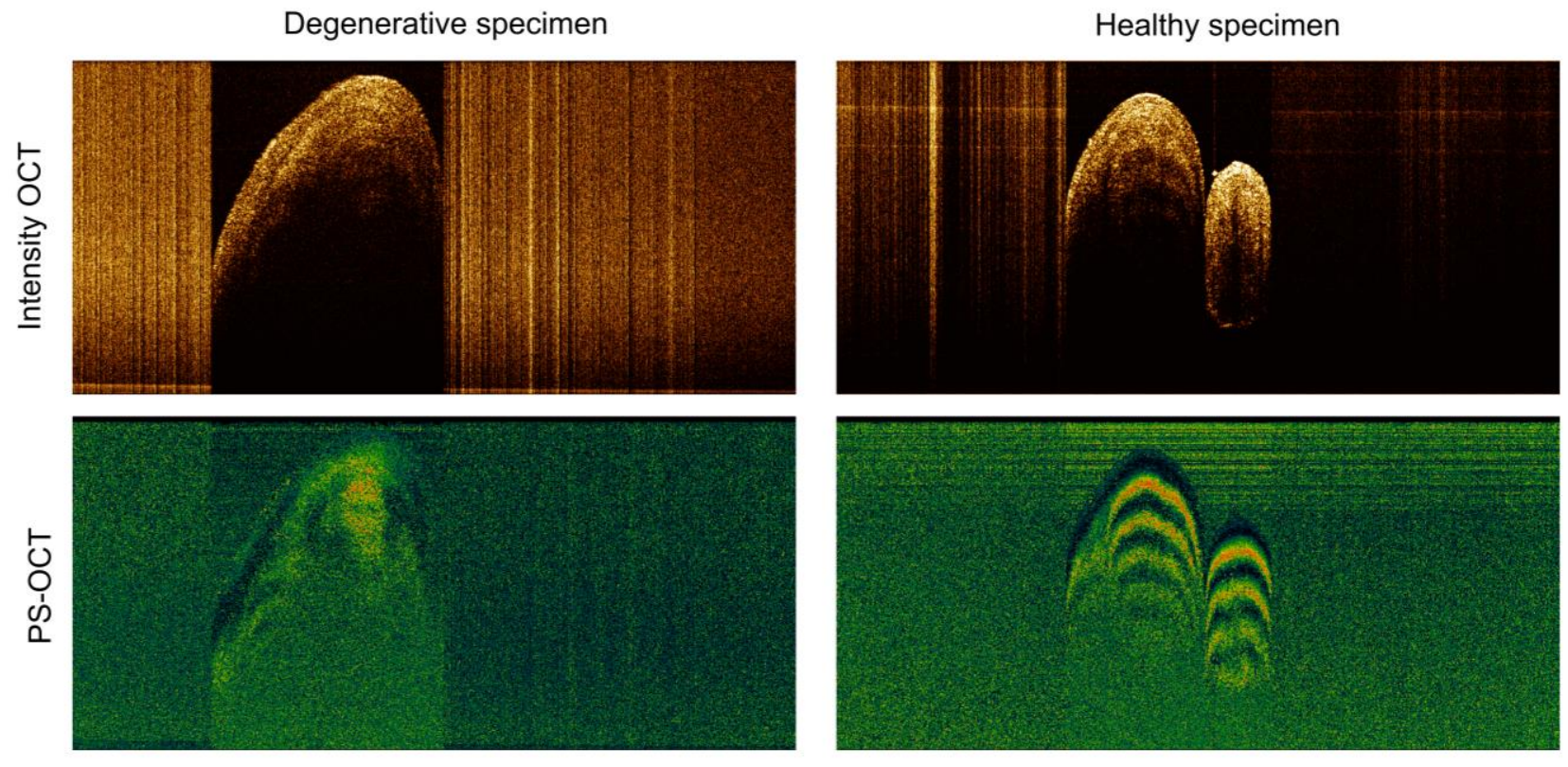

Figure 2. Comparison between a degenerative (left) and a healthy cord (right). In the first case, although phase differences are visualized, they lack periodicity produced by birefringent tissue.

The pattern exhibited by the different chords have been measured, obtaining the period of the bands for nine chords from five specimens. Applying Equation 1, the following birefringence values have been obtained:

Table 1. Birefringence values obtained for the 9 characterized specimens.

\begin{tabular}{|c|c|c|c|c|c|c|c|c|c|}
\hline $\begin{array}{c}\text { Individual } \\
\text { chords }\end{array}$ & $\mathbf{1}$ & $\mathbf{2}$ & $\mathbf{3}$ & $\mathbf{4}$ & $\mathbf{5}$ & $\mathbf{6}$ & $\mathbf{7}$ & $\mathbf{8}$ & $\mathbf{9}$ \\
\hline $\boldsymbol{\delta}$ & $2.36 \cdot 10^{-3}$ & $3.11 \cdot 10^{-3}$ & $3.11 \cdot 10^{-3}$ & $3.67 \cdot 10^{-3}$ & $2.93 \cdot 10^{-3}$ & $3.85 \cdot 10^{-3}$ & $3.64 \cdot 10^{-3}$ & $2.93 \cdot 10^{-3}$ & $2.44 \cdot 10^{-3}$ \\
\hline
\end{tabular}

In the case of degenerative chordae, the lack of bands in the PS-OCT indicates that the tissue does not present significant birefringence. The obtained birefringence values are similar to those reported by [8] for bovine tendon from $\delta=2.8 \cdot 10^{-3}$ to $\delta=3.7 \cdot 10^{-3}$, being this tissue also predominantly collagen, although it presents different density. On the other hand, the birefringence of mitral chordae tendineae has been previously measured in [6] by the method of de Sénarmont [10] obtaining of values from $\delta \approx 6 \cdot 10^{-4}$ to $\delta \approx 11 \cdot 10^{-4}$ with a light source at $546 \mathrm{~nm}$. According to Equation 1 , when these values are recalculated for a wavelength of $1325 \mathrm{~nm}$, they range from $\delta \approx 1.5 \cdot 10^{-3}$ to $\delta \approx 2.7 \cdot 10^{-3}$, that are similar to the results obtained in this work. 


\section{CONCLUSIONS}

Human chordae tendineae have been analyzed by the first time both with OCT and PS-OCT. The tomographic images allow the visualization of the core structure of the chords with a non-invasive and real-time procedure, providing the ability to characterize its morphology and structure. Furthermore, the PS-OCT system allows visualization and quantification of the birefringence patterns present in the samples under measurement. The obtained birefringence values correlate with literature. The birefringence values are related to the collagen distribution in the chords. In the case of degenerative chords, the birefringence of the sample is modified or even disappears due to the alteration of the composition of the degraded chord. Future work will be aimed to correlate the birefringence parameter with the core collagen density, so as to provide an accurate and useful tool for diagnosis within intraoperative interventions.

\section{ACKNOWLEDGEMENTS}

The authors thank the "Ministerio de Economía, Industria y Competitividad" (MINECO) for their support in this work under projects DA2TOI (FIS2010-19860), SENSA (TEC2016-76021-C2-2-R), the "Instituto de Salud Carlos III" (ISCIII) through projects FUSIODERM (DTS15/00238) and CIBERBBN and the co-financed by FEDER funds.

\section{REFERENCES}

[1] Millington-Sanders, C., Meir, A., Lawrence, L. and Stolinski, C.,"Structure of chordae tendineae in the left ventricle of the human heart," J Anat, 192(4), 573-81 (1998)

[2] Revuelta, J.M., García, R., Gaite, L., Val, F. and Garijo, F.,"Generation of chordae tendineae with polytetrafluoroethylene stents: Results of mitral valve chordae replacement in sheep" J Thorac Cardiov Sur, 103, 97:98-103 (1989)

[3] Icardo, J.M., Colvee, E. and Revuelta, J.M.,"Structural analysis of chordae tendineae in degenerative disease of the mitral valve," Int J Cardiol. 167(4), 1603-1609 (2012)

[4] Gabbay, U. and Yosefy, C., "The underlying causes of chordae tendineae rupture: A systematic review" Int J Cardiol, 143(2), 113-118 (2010)

[5] Bernal, J.M., Rabasa, J.M., Olalla, J.J., Carrión, M.F., Alonso, A. and Revuelta, J.M.,”Repair of chordae tendineae for rheumatic mitral valve disease. A twenty-year experience," J Thorac Cardiov Sur, 111, 211-7 (1996)

[6] Whittaker, P., Boughner, D.R., Perkins, D.G. and Canham, P.B.,'Quantitative structural analysis of collagen in chordae tendineae and its relation to floppy mitral valves and proteoglycan infiltration" Brit Heart J, 57(3), 264269 (1987)

[7] Otsu, N., "A Threshold Selection Method from Gray-Level Histograms," IEEE Sys Man Cyb 9(1), 62-66 (1979)

[8] de Boer, J.F., Milner, T.E., van Gemert, M.J. and Nelson, J.S.,’Two-dimensional birefringence imaging in biological tissue by polarization-sensitive optical coherence tomography," Opt Lett, 22(12), 934-936 (1997)

[9] Tearney, G.J., Brezinski, M.E., Southern, J.F., Bouma, B.E., Hee, M.R. and Fujimoto, J.G., 'Determination of the refractive index of highly scattering human tissue by optical coherence tomography," Opt Lett, 20(21), 2258-2260 (1995)

[10] Bennett H.S., "The microscopical investigation of biological materials with polarized light" in McClungJones R, ed. McClung's 'Handbook of microscopical technique', New York: PB Hoeber, 591-677 (1950) 